\title{
Forest Species Recognition using Color-based Features
}

\author{
P. L. Paula Filho, L. S. Oliveira, A. S. Britto Jr, R. Sabourin \\ Federal University of Parana, Department of Informatics, Curitiba, PR, Brazil \\ Pontifical Catholic University of Parana, PPGIA, Curitiba, PR, Brazil \\ Ecole de Technologie Superieure, Montreal, QC, Canada \\ lesoliveira@inf.ufpr.br
}

\begin{abstract}
In this work we address the problem of forest species recognition which is a very challenging task and has several potential applications in the wood industry. The first contribution of this work is a database composed of 22 different species of the Brazilian flora that has been carefully labeled by expert in wood anatomy. In addition, in this work we demonstrate through a series of comprehensive experiments that color-based features are quite useful to increase the discrimination power for this kind of application. Last but not least, we propose a segmentation approach so that an wood can be locally processed to mitigate the intra-class variability featured in some classes. Such an approach also brings important contribution to improve the final performance in terms of classification.
\end{abstract}

\section{Introduction}

The correct identification of forest species is of vital need for the wood industry and it has several different applications. In the recent years with the advent of globalization the safe trade of log and timber has become an important issue. An example of application that can save millions of dollars, in this case, would be to prevent frauds where a wood trader might mix a noble species with cheaper ones, or even try to export wood whose species is endangered.

Identifying an wood log or timber outside of its context (the forest) is not an easy task since one cannot count on flowers, fruits, and leaves. Therefore this task is performed by well trained specialists but few reach good accuracy in classification due to the time it takes for their training, hence they are not enough to meet the industry demands. Another factor to be taken into account is that the process of manual identification is rather time consuming which might lead to diversion of attention as it is a repetitive process and consequently may result in errors, which can become impracticable when checking cargo for export.
In this context, computer vision systems become very interesting. In the last decade, however, most of the applications of computer vision in the wood industry were related to quality control, grading, and defect detection $[2,8,5]$. Just recently, some authors began to use computer vision to classify forest species.

Khalid et al [6] present a system to recognize 20 different Malaysian forest species. A particularity of this method is that the wood samples were first boiled and then cut with a microtome into thin sections. The image acquisition was then performed with an industrial camera of high performance and a LED array lighting. The recognition process is based on a neural network trained with information extracted from the gray-level co-occurrence matrix (GLCM). The database used in their experiments contains $1,753 \mathrm{im}-$ ages for training and only 196 for testing. They report a recognition rates of $95 \%$. A drawback of this strategy is the expensive acquisition protocol which makes it unfeasible for real applications.

Tou et al [9] reported two experiments to classify forest species which also use GLCM features to train a neural network classifier. A database of 360 images taken at the Center for Artificial Intelligence and Robotics (CAIRO) was used to perform the experiments. The authors report recognition rates ranging from $60 \%$ to $72 \%$ for five different forest species.

The number of systems that effectively address the recognition of forest species is small due to the following aspects [6]: a) difficulty in obtaining a labeled database, b) lack of proven techniques for recognition of forest species, c) use of expensive equipment, and d) lack of experts on classification in developing countries. Regarding the species recognition of the Brazilian flora, little bibliographic material has been developed, and the majority of the works differentiates few species and uses techniques of near infrared spectroscopy [7].

The main contribution of this paper is two-fold. Firstly, we increased the first version [3] of the database of Brazilian forest species by adding 11 new classes. This second ver- 
sion now contains 22 different species which were labeled by specialists in the Laboratory of Wood Anatomy of the Federal University of Parana (UFPR). Secondly, we investigated the use of color-based features to improve the recognition performance for this task. By analyzing different color models we have defined a set of color-based features that uses information from three different color spaces. Besides, we also proposed a segmentation strategy to deal with the great intra-class variability observed in some species. A comprehensive series of experiments shows that the proposed strategies can improve the results in about $20 \%$ for this data set when compared with the traditional GLCM features.

\section{Database}

The database used in this work is composed of 1270 images of 22 different forest species which were acquired in the Laboratory of Wood Anatomy of the UFPR (Federal University of Parana). It was divided into 402, 231, and 619 images for training, validation, and testing, respectively. The class distribution is reported in Table 1 and some samples are depicted in Figure 1.

Table 1. Species in the Database

\begin{tabular}{|c|c|c|c|c|c|}
\hline Class & Description & $\#$ & Class & Description & \# \\
\hline 1 & $\begin{array}{l}\text { Imbuia } \\
\text { Ocotea porosaa }\end{array}$ & 16 & 12 & $\begin{array}{l}\text { Uva do Japão } \\
\text { Hovenia Dulcis T. }\end{array}$ & 49 \\
\hline 2 & $\begin{array}{l}\text { Roxinho } \\
\text { Peltogyne sp }\end{array}$ & 228 & 13 & $\begin{array}{l}\text { Ipê } \\
\text { Tabebuia sp }\end{array}$ & 66 \\
\hline 3 & $\begin{array}{l}\text { Pinus } \\
\text { Pinus sp }\end{array}$ & 47 & 14 & $\begin{array}{l}\text { Bracatinga } \\
\text { Mimosa S. B. }\end{array}$ & 45 \\
\hline 4 & $\begin{array}{l}\text { Massaranduba } \\
\text { Manilkara spp }\end{array}$ & 52 & 15 & $\begin{array}{l}\text { Andiroba } \\
\text { Carapa G. Aub }\end{array}$ & 45 \\
\hline 5 & $\begin{array}{l}\text { Pau Cetim } \\
\text { Aspidosperma sp }\end{array}$ & 74 & 16 & $\begin{array}{l}\text { Amapá } \\
\text { Brosimum P. Ducke }\end{array}$ & 57 \\
\hline 6 & $\begin{array}{l}\text { Louro } \\
\text { Laurus nobilis }\end{array}$ & 92 & 17 & $\begin{array}{l}\text { Cumaru } \\
\text { Dipteryx sp }\end{array}$ & 31 \\
\hline 7 & $\begin{array}{l}\text { Cedro } \\
\text { Cedrela F. V. }\end{array}$ & 32 & 18 & $\begin{array}{l}\text { Cedrinho } \\
\text { Erisma U. Warm }\end{array}$ & 84 \\
\hline 8 & $\begin{array}{l}\text { Acrocarpus } \\
\text { Acrocarpus F. }\end{array}$ & 26 & 19 & $\begin{array}{l}\text { Castanheira } \\
\text { Bertolethia E. H. B. }\end{array}$ & 57 \\
\hline 9 & $\begin{array}{l}\text { Frejo } \\
\text { Cordia G. H. }\end{array}$ & 60 & 20 & $\begin{array}{l}\text { Araucária } \\
\text { Araucaria A. }\end{array}$ & 40 \\
\hline 10 & $\begin{array}{l}\text { Grevilea } \\
\text { Grevillea R. A. }\end{array}$ & 49 & 21 & $\begin{array}{l}\text { Mogno } \\
\text { Swietenia M. K. }\end{array}$ & 24 \\
\hline 11 & $\begin{array}{l}\text { Pau Amarelo } \\
\text { Euxylophora P. H.r }\end{array}$ & 48 & 22 & $\begin{array}{l}\text { Jatoba } \\
\text { Hymenaea C. L. }\end{array}$ & 48 \\
\hline
\end{tabular}

In order to build this dataset two main criteria were adopted: The first one was the availability of labeled samples in the laboratory while the second was the level of confusion when the species were classified by human experts. Thus we selected mostly those species that normally create doubts in their classification to the naked eye.

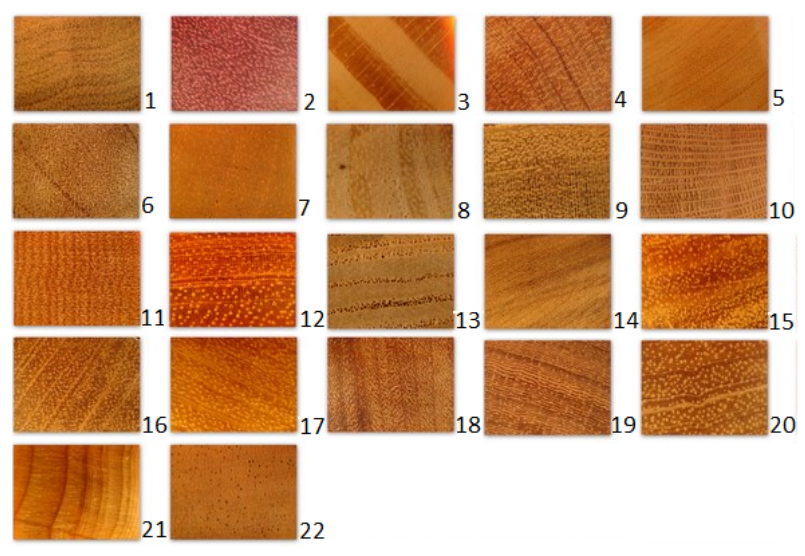

Figure 1. Samples of the database

A protocol was defined for the image acquisition to ensure standardized distance and lighting. The camera was fixed into a base along with two halogen lamps so that we would have homogenous light. A low-cost digital camera (SONY DSC T20) with both macro mode and auto-focus enabled was used to acquire the images. The distance between the camera lens and the wood samples was 20 millimeters. The images were obtained with a resolution of $3,264 \times 2,448$ pixels. It is important to mention that the only technique applied to the wood sample before acquisition was sanding. This technique, along with a low-cost camera, requires no structure in the field and alike more complex techniques such as wood boiling, microtome use, it also ensure a good visualization of the wood structures.

After some visual analysis, we have noticed that some species have a great intra-class variability, which has a considerable impact on the recognition performance. As discussed in [3], this variability can be either something inherent to the class, like the different colors observed in Figure $2 \mathrm{a}$ or something produced by the cutting apparatus, like the diagonal marks in Figure 2b.
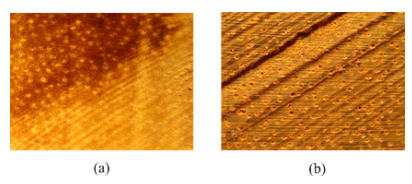

Figure 2. Intra-class variability

The way we found to cope with this variability was to split the images into smaller images so that more homogeneous images could be generated. In this way, during testing the images would be equally segmented into smaller images and the final decision would be produced by some combination method such as voting, average, etc. After 
some experiments, we found out that segmenting the original images into 25 pieces of $130 \times 98$ pixels produced good results. Having this in mind, after this segmentation, the training, validation, and testing sets contain 10,501, 5,776, and 15,476 images, respectively.

\section{Features}

As stated before few works in the literature have addressed the problem of forest species recognition. Those working in this field [6,9], though, generally consider GLCM and its variations to extract features. This makes sense since this problem can be formulated as a texture recognition problem. Besides texture, in this work we argue that color could be very useful to improve recognition rates. In the next subsections we describe both color-based and the GLCM features used in this work.

\subsection{Color}

When discussing color image processing, one of the most employed features is the color histogram, which is a representation of the distribution of colors in an image. Considering a digital image, it is basically the number of pixels for each color covered by the color space. Among the most used color spaces for feature extraction we can cite the RGB (Red, Green, Blue) and HSI (Hue, Saturation, Intensity). Several works in the literature have successfully applied color features to different pattern recognition problems. In the context of wood classification, we can cite the works of Kauppinen [5] and Cavalin et al [1] who had used the percentile values extracted from an RGB color histograms for wood grading.

In this work, however, we have used a more perceptive approach to define the color features. The choice of which features to be used was done by an empirical analysis of histograms collected from color channels of different color spaces. The idea was to choose those channels that maximize the inter-class and minimize the intra-class variance, respectively. Of course this is not an easy task, but it is very important to define an initial feature set with a good discriminative power. By the end of this analysis, we have chosen to use the following color channels: $G$ (green) from $\mathrm{RGB}, \mathrm{S}$ (saturation) from HSV, and L (luminance) from CIELUV.

We also have noticed that different classes have the information concentrated in certain zones of the histogram, as depicted in Figure 3. To take advantage of this fact, we decided to implement a local analysis by dividing the histogram into two zones and then extracting one feature vector from each zone. The first zone covers those pixels from 0 to 200 while the second zone covers those from 201 to 255 .

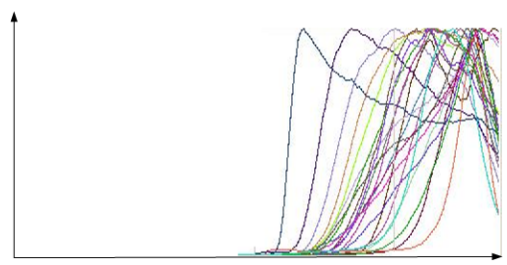

Figure 3. Analysis of the color channels.

From each histogram three characteristics based on first order statistics were calculated: average, kurtosis, and obliquity. These features were normalized according to min-max rule. Considering the three color channels mentioned before and the zoning strategy used, in the end we have a feature vector composed of 18 (3 features $\times 3$ histograms $\times 2$ zones) components. It is important to remark that this proposed feature set surpassed the results achieved by the traditional RGB histograms used in [5].

\subsection{Co-Occurrence Matrix}

Among the statistical techniques of texture recognition, the Gray-Level Co-occurrence Matrix (GLCM) has been one of the most used and successful ones. This technique consists of statistical experiments conducted on how a certain level of gray occurs on other levels of gray [10]. It intuitively provides measures of properties such as smoothness, coarseness, and regularity. Haralick [4], the precursor of this technique suggested a set of 14 characteristics, but most of works in the literature consider a subset of these descriptors. In our case, we used the following six: Energy, Contrast, Entropy, Homogeneity, Maximum Likelihood and 3rd Order Moment.

By definition, a GLCM is the joint probability occurrence of graylevel $i$ and $j$ within a defined spatial relation in an image. That spatial relation is defined in terms of a distance $d$ and an angle $\theta$. Given a GLCM, some statistical information can be extract from it. Assuming that $\mathrm{Ng}$ is the graylevel depth, and $p(i, j)$ is the probability of the co-occurrence of graylevel $i$ and graylevel $j$ observing consecutive pixels at distance $d$ and angle $\theta$, to describe wood texture.

In our experiments we have tried different values for $d$ as well as different angles. The best setup we have found is $d=1$ and $\theta=[0,45,90,135]$. Considering the six descriptor aforementioned, in the end we have a feature vector of 24 components.

\section{Experiments}

To evaluate the performance of the aforementioned feature sets we have used an MLP (Multi-Layer Perceptron) 
trained with the gradient descent applied to a sum-ofsquares error function. The transfer function employed is the standard sigmoid function. In order to monitor the generalization performance during learning and terminate the algorithm when there is no longer an improvement, we have used the method of validation. Such a method takes into account a validation set, which is not used for learning, to measure the generalization performance of the network. During learning, the performance of the network on the training set will continue to improve, but its performance on the validation set will only improve to a point, where the network starts to overfit the training set, that the learning algorithm is terminated. The parameters of the networks were set empirically.

To better assess the impact of the proposed feature sets we have trained three different classifiers so that both feature sets could be assessed independently. In the first experiment we have used a classifier trained with GLCM-based features. In the second experiment, the classifier used was the one trained with color-based features. Finally, for the last experiment we merged both feature sets so that the classifier was trained with a 40-dimensional feature vector. In all experiments the neural networks has 22 outputs. As mentioned before, in this work we proposed the segmentation of the image into 25 pieces to deal with the intra-class variability. Therefore, each testing image is segmented into 25 pieces which go through the feature extraction and classification steps. Then, the 25 decisions produced by the classifier are combined using a voting scheme to yield a final decision. Table 2 summarizes all the experiments.

Table 2. Summary of the experiments

\begin{tabular}{lccc}
\hline \multicolumn{1}{c}{ Strategy } & GLCM & Color & Color+GLCM \\
\hline Full Image & 43.3 & 61.5 & 66.3 \\
Voting & 57.8 & 78.2 & 80.8 \\
\hline
\end{tabular}

As we can observe in Table 2 both strategies proposed in this paper bring some improvement to the problem of forest species recognition. The color features, which were defined based on visual observations of the several color channels of different color spaces, brought an considerable improvement of more than $20 \%$ when compared to the traditional GLCM (texture-based) features. This supports our initial hypothesis and motivates us to explore the color-based features with more interest. Besides, the use of the proposed segmentation scheme also brought an important contribution in terms of performance. From Table 2, it can be observed that this segmentation strategy can solve several confusions, thus achieving a performance of $78 \%$ against $61 \%$ of the system based on full images (no segmentation). A final consideration regards the use of the GLCM feature set. In spite of the fact that it was surpassed by the color-features in our experiments, we can state that they are complementary in some cases. The experiments show that using both feature sets the performance were improved in about $5 \%$ and $3 \%$ for full images and segmented images, respectively.

\section{Conclusions and Future Works}

In this work we have discussed the problem of forest species recognition which is a very challenging problem and has an enormous potential for real industrial applications. We have demonstrated through a series of comprehensive experiments that the color-based features can improve the results achieved by the traditional texture-based classifiers. Besides, the proposed segmentation scheme proved very useful in reducing the confusion created because of the intra-class variation featured by some species. In this work we have used 25 fixed zones, but different number of zones can be used to train different classifiers where the results can be further combined. In the light of the difficulties of building a reliable database in this field, we believe that the database proposed in this work can be very useful for further investigations in this field. As future works, we are working on new features and also new classification strategies as the classes in the database tend to increase.

\section{References}

[1] P. Cavalin, L. S. Oliveira, A. L. Koerich, and A. S. BrittoJr. Wood defect detection using grayscale images and an optimized feature set. In 32nd Annual Conf. of the IEEE Industrial Electronics Society, pages 3408-3412, 2006.

[2] R. Conners, D. Kline, P. Araman, and T. Drayer. Machine vision technology for the forest products industry. IEEE Computer, 30:43, 1997.

[3] P. L. P. Filho, L. S. Oliveira, and A. S. Britto-Jr. A database for forest species recognition. In XXII Brazilian Symposium on Computer Graphics and Image Processing, 2009.

[4] R. M. Haralick. Statistical and structural approaches to texture. Proceedings of IEEE, 67(5), 1979.

[5] H. Kauppinen. A two stage defect recognition method for parquet slab grading. In Procs of the International Conference on Pattern Recognition, pages 803-806, 2000.

[6] M. Khalid, E. L. Y. Lee, R. Yusof, and M. Nadaraj. Design of an intelligent wood species recognition system. IJSSST, 9(3), 2008.

[7] G. I. B. Muniz, M. E. Carneiro, and T. Silva. The use of the infrared and multivariate analytic for identification of species of timber. IV Congresso Florestal Latinoamericano, 2008.

[8] L. Thomas and L. Milli. A robust gm-estimator for the automated detection of external defects on barked hardwood logs and stems. IEEE Trans on Sig. Proc., 55:3568, 2007.

[9] J. Y. Tou, P. Y. Lau, and Y. H. Tay. Computer visionbased wood recognition system. Proceedings of International Workshop on Advanced Image Technology, 2007.

[10] M. Tuceryan and A. K. Jain. Texture analysis. In The Handbook of Pattern Recognition and Computer Vision. 1998. 